\title{
Differential-Linear Attacks Against the Stream Cipher Phelix ${ }^{\star}$
}

\author{
Hongjun $\mathrm{Wu}$ and Bart Preneel \\ Katholieke Universiteit Leuven, ESAT/SCD-COSIC \\ Kasteelpark Arenberg 10, B-3001 Leuven-Heverlee, Belgium \\ \{wu.hongjun, bart.preneel\}@esat.kuleuven.be
}

\begin{abstract}
The previous key recovery attacks against Helix obtain the key with about $2^{88}$ operations using chosen nonces (reusing nonce) and about 1000 adaptively chosen plaintext words (or $2^{35.6}$ chosen plaintext words). The stream cipher Phelix is the strengthened version of Helix. In this paper we apply the differential-linear cryptanalysis to recover the key of Phelix. With $2^{34}$ chosen nonces and $2^{37}$ chosen plaintext words, the key of Phelix can be recovered with about $2^{41.5}$ operations.
\end{abstract}

\section{Introduction}

Phelix [5] is a fast stream cipher with an embedded authentication mechanism. It is one of the focus ciphers (both software and hardware) of the ECRYPT eSTREAM project. Phelix is a strengthened version of the stream cipher Helix [1].

Muller has applied differential attack to Helix 2. He showed that the key of Helix can be recovered faster than by brute force if the attacker can force the initialization vectors to be used more than once. The attack requires about $2^{12}$ adaptively chosen plaintext words and $2^{88}$ operations. Paul and Preneel reduced the number of adaptively chosen plaintext words by a factor of at least 3 [4]. Later Paul and Preneel showed that $2^{35.6}$ chosen plaintext words can be used instead of adaptively chosen plaintexts 3 . All these key recovery attacks against Helix require about $2^{88}$ operations.

Phelix was designed and submitted to the ECRYPT eSTREAM project in 2005. The output function of Helix has been changed so that a larger plaintext diffusion can be achieved in Phelix. The Phelix designers claimed that Phelix is able to resist a differential key recovery attack even if the nonce is reused: "We claim, however, that even in such a case (referring to nonce reuse) it remains infeasible to recover the key" [5].

In this paper, we apply differential-linear cryptanalysis to Phelix assuming nonce reuse (this corresponds to a chosen nonce attack). We show that the key of Phelix can be recovered with a low complexity: $2^{37}$ chosen plaintext words and $2^{41.5}$ operations. Although the Phelix designers did expect that Phelix would

\footnotetext{
* This work was supported in part by the Concerted Research Action (GOA) Ambiorics 2005/11 of the Flemish Government and in part by the European Commission through the IST Programme under Contract IST-2002-507932 ECRYPT.
} 
loose most of its security properties when the nonce is reused, this paper shows that Phelix is completely insecure in such a setting.

This paper is organized as follows. In Sect. 2, we illustrate the operations of Phelix. Section 3 analyzes how the addend bits affect the differential distribution. Section 4 describes a basic differential key recovery attack on Phelix. The improved attack is given in Sect. 5. We discuss how to strengthen Phelix in Sect. 6. Section 7 concludes this paper.

\section{The Stream Cipher Phelix}

In this section, we only consider the encryption algorithm of Phelix. The full description of Phelix is given in [5]. The key size and nonce size of Phelix are 256 bits and 128 bits, respectively. The designers claim that there is no attack against Phelix with less than $2^{128}$ operations.

Phelix updates fives 32-bit words: $Z_{0}, Z_{1}, Z_{2}, Z_{3}$ and $Z_{4}$. At the $i$ th step, two secret 32 -bit words $X_{i, 0}, X_{i, 1}$ and one 32 -bit plaintext word $P_{i}$ are applied to update the internal states. One 32 -bit keystream word $S_{i}$ is generated and is used to encrypt the plaintext $P_{i}$. Note that the plaintext is used to update the internal state so that the authentication can be performed. The word $X_{i, 0}$ is related to the key, and the word $X_{i, 1}$ is related to the key and nonce in a very simple way. Recovering any $X_{i, 0}$ and $X_{i, 1}$ implies recovering part of the key. One step of Phelix is given in Fig. 1.

\section{The Differential Propagation of Addition}

In this section, we study how the addend bits affect the differential propagation. The importance of this study is that it shows that the values of the addend bits can be determined by observing the differential distribution of the sum.

Theorem 1. Denote $\phi_{i}$ as the $i$ th least significant bit of $\phi$. Suppose two positive $m$-bit integers $\phi$ and $\phi^{\prime}$ differ only at the $n$th least significant bit position $(\phi \oplus$ $\left.\phi^{\prime}=2^{n}\right)$. Let $\beta$ be an $m$-bit random integer $(m$ is much larger than $n)$. Let $\psi=\phi+\beta$ and $\psi^{\prime}=\phi^{\prime}+\beta$. For $\beta_{n}=0$, denote the probability that $\psi_{n+i}=\psi_{n+i}^{\prime}$ as $p_{n+i, 0}$. For $\beta_{n}=1$, denote the probability that $\psi_{n+i}=\psi_{n+i}^{\prime}$ as $p_{n+i, 1}$. Then the difference $\Delta p_{n+i}=p_{n+i, 0}-p_{n+i, 1}=2^{-n-i+1}(i>0)$.

Theorem 1 can be proved easily if we consider the bias in the carry bits. We omit the proof here. In Theorem 1, the bias of the differential distribution decreases quickly as the value of $n$ increases. We need another differential property that produces difference with a large bias even for large $n$. Before introducing that property, we give the following lemma from [6].

Lemma 1 . Denote $u$ and $v$ as two random and independent $n$-bit integers. Let $c_{n}=(u+v) \gg n$, where $c_{n}$ denotes the carry bit at the $n$th least significant bit position. Denote the most significant bit of $u$ as $u_{n-1}$. Then $\operatorname{Pr}\left(c_{n} \oplus u_{n-1}=0\right)=\frac{3}{4}$.

The large bias of the differential distribution for large $n$ is given below. 


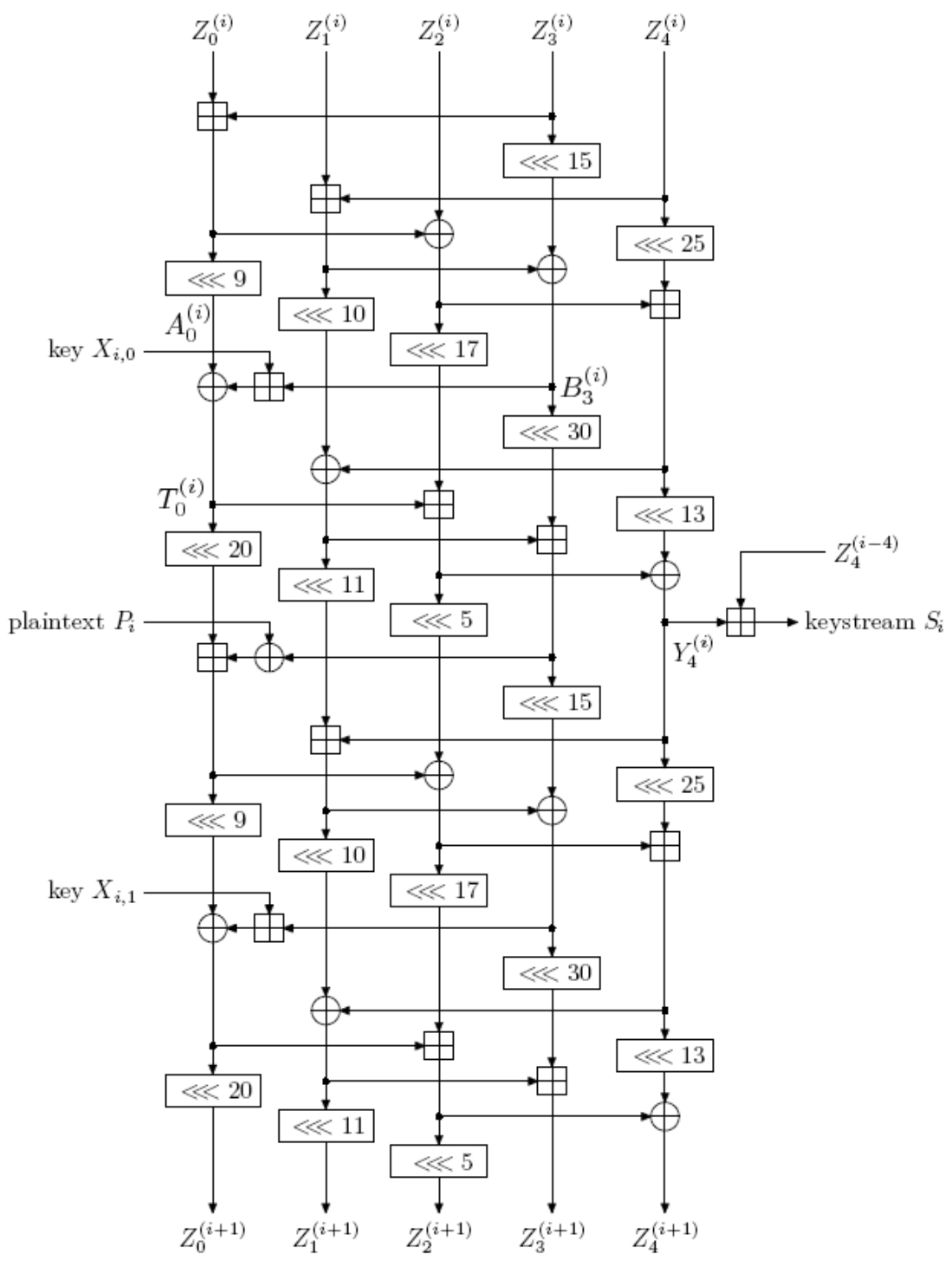

Fig. 1. One block of Phelix [5]

Theorem 2. Denote $\phi_{i}$ as the $i$ th least significant bit of $\phi$. Suppose two positive $m$-bit integers $\phi$ and $\phi^{\prime}$ differ only at the $n$th least significant bit position $\left(\phi \oplus \phi^{\prime}=2^{n}\right)$. Let $\beta$ be an $m$-bit random integer $(m$ is much larger than $n)$. Let $\psi=\phi+\beta$ and $\psi^{\prime}=\phi^{\prime}+\beta$. For $\beta_{n} \oplus \beta_{n-1}=0$, denote the probability that $\psi_{n+i}=\psi_{n+i}^{\prime}$ as $\bar{p}_{n+i, 0}$. For $\beta_{n} \oplus \beta_{n-1}=1$, denote the probability that $\psi_{n+i}=\psi_{n+i}^{\prime}$ as $\bar{p}_{n+i, 1}$. Then the difference $\Delta \bar{p}_{n+i}=\bar{p}_{n+i, 0}-\bar{p}_{n+i, 1}=2^{-i}(i>0)$. 
Proof. Denote the carry bit at the $i$ th least significant bit position in $\psi=\phi+\beta$ as $c_{i}$, and that in $\psi^{\prime}=\phi^{\prime}+\beta$ as $c_{i}^{\prime}$. Note that $c_{n}^{\prime}=c_{n}$, thus $c_{n}^{\prime} \oplus \beta_{n}=c_{n} \oplus \beta_{n}$. When $c_{n}^{\prime} \oplus \beta_{n}=c_{n} \oplus \beta_{n}=0$, we know that $\psi \oplus \psi^{\prime}=2^{n}$ with probability 1, i.e., $\psi_{n+i}=\psi_{n+i}^{\prime}$ with probability 1 for $i>0$. When $c_{n}^{\prime} \oplus \beta_{n}=c_{n} \oplus \beta_{n}=1$, by induction we obtain that $\psi_{n+i}=\psi_{n+i}^{\prime}$ with probability $1-2^{-i+1}$ for $i>0$. According to Lemma 1, we know that $c_{n} \oplus \beta_{n-1}=0$ with probability $\frac{3}{4}$. If $\beta_{n} \oplus \beta_{n-1}=0$, then $c_{n} \oplus \beta_{n}=0$ with probability $\frac{3}{4}$, thus $\bar{p}_{n+i, 0}=\frac{3}{4} \times 1+\frac{1}{4} \times\left(1-2^{-i+1}\right)=$ $1-\frac{1}{4} \times 2^{-i+1}$. If $\beta_{n} \oplus \beta_{n-1}=1$, then $c_{n} \oplus \beta_{n}=0$ with probability $\frac{1}{4}$, thus $\bar{p}_{n+i, 1}=\frac{1}{4} \times 1+\frac{3}{4} \times\left(1-2^{-i+1}\right)=1-\frac{3}{4} \times 2^{-i+1}$. Then the difference $\Delta \bar{p}_{n+i}=\bar{p}_{n+i, 0}-\bar{p}_{n+i, 1}=2^{-i}$ for $i>0$.

The above two theorems provide the guidelines to recover the key of Phelix. However, these two theorems deal with the ideal cases in which there is only one bit difference between $\phi$ and $\phi^{\prime}$, and $\beta$ is assumed to be random. In the attacks, we deal with the complicated situation where each bit of $\phi \oplus \phi^{\prime}$ is biased, and $\beta$ is a fixed integer. The value of each bit of $\beta$ will affect the distribution of the higher order bits of $(\phi+\beta) \oplus\left(\phi^{\prime}+\beta\right)$ in a complicated way. In order to simplify the analysis, we will use simulations to obtain these relations in the attacks.

\section{A Basic Key Recovery Attack on Phelix}

We will first investigate the differential propagation in Phelix. Then we show how to recover the key of Phelix by observing the differential distribution of the keystream.

\subsection{The Bias in the Differential Distribution of the Keystream}

Assume an attacker can choose an arbitrary value for the nonce, then a nonce can be used more than once. We introduce one-bit difference into the plaintext at the $i$ th step, i.e., $P_{i} \neq P_{i}^{\prime}$, and $P_{i} \oplus P_{i}^{\prime}=2^{n}(0 \leq n \leq 31)$. Then we analyze the difference between $B_{3}^{(i+1)}$ and $B_{3}^{\prime(i+1)}$ (as indicated in Fig. 1). If all the carry bits are 0 (replacing all the additions with XORs), then the differences only appear at the 9 th, 11th, 13 th, 15 th and 17 th least significant bits between $B_{3}^{(i+1)}$ and $B_{3}^{\prime(i+1)}$. Because of the carry bits, the differential distribution becomes complicated. We run the simulation and use the randomly generated $Y_{k}^{(i)}(4 \geq k \geq 0)$, $P_{i}, X_{i, 1}$ in the simulation. With $2^{30}$ plaintext pairs, we obtain the distribution of $B_{3}^{(i+1)} \oplus B_{3}^{\prime(i+1)}$ in Table 1 .

From Table 1, we see that the distribution of $B_{3}^{(i+1)} \oplus B_{3}^{\prime(i+1)}$ is heavily biased. For example, $B_{3}^{(i+1), 8}=B_{3}^{\prime(i+1), 8}$ with probability close to 1 , while $B_{3}^{(i+1), 9}=$ $B_{3}^{\prime(i+1), 9}$ with probability close to 0 . Note that $T_{0}^{(i+1)}=A_{0}^{(i+1)} \oplus\left(B_{3}^{(i+1)}+\right.$ $\left.X_{i+1,0}\right)$, according to Theorem 2 , the distribution of $T_{0}^{(i+1)} \oplus T_{0}^{\prime(i+1)}$ will be affected by the value of $X_{i+1,0}^{8} \oplus X_{i+1,0}^{9}$, thus the distribution of $B_{i+1} \oplus B_{i+1}^{\prime}$ will be affected by the value of $X_{i, 0}^{8} \oplus X_{i, 0}^{9}$. By observing the distribution of $S_{i+1} \oplus S_{i+1}^{\prime}$, it may be possible to determine the value of $X_{i, 0}^{8} \oplus X_{i, 0}^{9}$. Shifting 
Table 1. The probability that $B_{3}^{(i+1), j} \oplus B_{3}^{\prime(i+1), j}=0$ for $P_{i} \oplus P_{i}^{\prime}=1$

\begin{tabular}{|c|c||c|c||c|c||c|c|}
\hline$j$ & $p$ & $j$ & $p$ & $j$ & $p$ & $j$ & $p$ \\
\hline 0 & 0.9997 & 8 & 1.0000 & 16 & 0.5001 & 24 & 0.9161 \\
1 & 0.9998 & 9 & 0.0000 & 17 & 0.4348 & 25 & 0.9470 \\
2 & 0.9999 & 10 & 0.5000 & 18 & 0.5000 & 26 & 0.9673 \\
3 & 0.9999 & 11 & 0.4375 & 19 & 0.5486 & 27 & 0.9803 \\
4 & 1.0000 & 12 & 0.5000 & 20 & 0.6366 & 28 & 0.9883 \\
5 & 1.0000 & 13 & 0.4492 & 21 & 0.7283 & 29 & 0.9931 \\
6 & 1.0000 & 14 & 0.5000 & 22 & 0.8083 & 30 & 0.9960 \\
7 & 1.0000 & 15 & 0.4273 & 23 & 0.8708 & 31 & 0.9977 \\
\hline
\end{tabular}

the one-bit difference between $P_{i}$ and $P_{i}^{\prime}$, we may determine other values of $X_{i, 0}^{j+1} \oplus X_{i, 0}^{j}$ for $0 \leq j \leq 30$, and recover in this way the key $X_{i, 0}$. After recovering eight consecutive $X_{i, 0}$ values, the 256 -bit key can be found immediately.

The above analysis gives a brief idea of the attack. However, the actual attacks are quite complicated due to the interference of many differences. It is very tedious to derive exactly how the distribution of $S_{i+1} \oplus S_{i+1}^{\prime}$ is affected by the value of $X_{i+1,0}^{j+1} \oplus X_{i+1,0}^{j}$. On the other hand, it is easy to search for the relation with simulations. In the following, we carried out a simulation to find the relation between the value of $X_{i+1,0}^{j+1} \oplus X_{i+1,0}^{j}$ and the distribution of $S_{i+1} \oplus S_{i+1}^{\prime}$.

Let two plaintexts differ only in the $i$ th word, and $P_{i} \oplus P_{i}^{\prime}=1$. We use the randomly generated $Y_{k}^{(i)}(4 \geq k \geq 0), P_{i}, X_{i, 1}, X_{i+1,0}, Z_{4}^{(i-3)}$ in the simulation. Denote with $p_{j, 0}^{n}$ the probability that $S_{i+1}^{n} \oplus S_{i+1}^{\prime n}=0$ when $X_{i, 0}^{j+1} \oplus X_{i, 0}^{j}=0$. And denote $p_{j, 1}^{n}$ as the probability that $S_{i+1}^{n} \oplus S_{i+1}^{\prime n}=0$ when $X_{i, 0}^{j+1} \oplus X_{i, 0}^{j}=1$. Let $\Delta \tilde{p}_{j}^{n}=\left(p_{j, 0}^{n}-p_{j, 1}^{n}\right) \times \frac{N}{\sigma}$, where $N$ denotes the number of plaintext pairs, and $\sigma=\frac{\sqrt{N}}{2}$. Assume that the values of $p_{j, 0}^{n}$ and $p_{j, 1}^{n}$ are close to $\frac{1}{2}$. If $\Delta \tilde{p}_{j}^{n}>4$, the difference between $p_{j, 0}^{n}$ and $p_{j, 1}^{n}$ is larger than $4 \sigma$, hence the value of $X_{i, 0}^{j+1} \oplus X_{i, 0}^{j}$ can be determined correctly with high probability. For every value of the two bits $X_{i, 0}^{j+1}$ and $X_{i, 0}^{j}$, we use $2^{28}$ pairs to generate $S_{i+1} \oplus S_{i+1}^{\prime}$, then compute $p_{j, 0}^{n}$ and $p_{j, 1}^{n}$. Thus $N=2^{29}$, and $\sigma=2^{13.5}$. We list the large values of $\Delta \tilde{p}_{j}^{n}$ below:

For $j=9, \Delta \tilde{p}_{9}^{13}=55.7$.

For $j=10, \Delta \tilde{p}_{10}^{13}=133.9$.

For $j=14, \Delta \tilde{p}_{14}^{17}=51.5$.

For $j=15, \Delta \tilde{p}_{15}^{19}=-9.1, \Delta \tilde{p}_{15}^{22}=14.9, \Delta \tilde{p}_{15}^{23}=-15.7$.

For $j=16, \Delta \tilde{p}_{16}^{19}=-50.8, \Delta \tilde{p}_{16}^{21}=62.0, \Delta \tilde{p}_{16}^{22}=97.7, \Delta \tilde{p}_{16}^{23}=-106.6$, $\Delta \tilde{p}_{16}^{25}=11.8, \Delta \tilde{p}_{16}^{26}=16.0, \Delta \tilde{p}_{16}^{27}=-17.4$.

For $j=17, \Delta \tilde{p}_{17}^{21}=77.4, \Delta \tilde{p}_{17}^{22}=145.3, \Delta \tilde{p}_{17}^{23}=-171.6, \Delta \tilde{p}_{17}^{25}=12.3$, $\Delta \tilde{p}_{17}^{26}=28.5, \Delta \tilde{p}_{17}^{27}=-30.4$.

For $j=18, \Delta \tilde{p}_{18}^{21}=80.2, \Delta \tilde{p}_{18}^{22}=179.7, \Delta \tilde{p}_{18}^{23}=-241.7, \Delta \tilde{p}_{18}^{26}=32.8$, $\Delta \tilde{p}_{18}^{27}=-43.7$. 
For $j=19, \Delta \tilde{p}_{19}^{22}=139.6, \Delta \tilde{p}_{19}^{23}=-220.6, \Delta \tilde{p}_{19}^{26}=19.0, \Delta \tilde{p}_{19}^{27}=-46.5$.

For $j=20, \Delta \tilde{p}_{20}^{23}=-156.7, \Delta \tilde{p}_{20}^{25}=-5.7, \Delta \tilde{p}_{20}^{26}=18.3, \Delta \tilde{p}_{20}^{27}=-30.6$.

For $j=21, \Delta \tilde{p}_{21}^{25}=-6.8, \Delta \tilde{p}_{21}^{26}=9.5, \Delta \tilde{p}_{20}^{27}=-28.5$.

The data given above show that the distribution of $S_{i+1} \oplus S_{i+1}^{\prime}$ is strongly affected by the value of $X_{i+1,0}^{j+1} \oplus X_{i+1,0}^{j}$.

\subsection{Recovering the Key}

Note that in the above analysis, when we deal with a particular $X_{i+1,0}^{j+1} \oplus X_{i+1,0}^{j}$, the other bits of $X_{i+1,0}$ are random. In the key recovery attack, the value of $X_{i+1,0}$ is fixed, so we need to consider the interference between the bits $X_{i+1,0}^{j+1} \oplus$ $X_{i+1,0}^{j}$.

We notice that there are many large biases related to $S_{i+1}^{23} \oplus S_{i+1}^{23}$. However, the values of $X_{i+1,0}^{j+1} \oplus X_{i+1,0}^{j}(15 \leq j \leq 20)$ all have a significant effect on the distribution of $S_{i+1}^{23} \oplus S_{i+1}^{23}$. It is thus a bit complicated to determine the values of $X_{i+1,0}^{j+1} \oplus X_{i+1,0}^{j}(15 \leq j \leq 20)$.

In the following, we consider the bit $S_{i+1}^{17} \oplus S_{i+1}^{\prime 17}$. Its distribution is dominated by the value of $X_{i+1,0}^{15} \oplus X_{i+1,0}^{14}$. For every value of the two bits $X_{i+1,0}^{15}$ and $X_{i+1,0}^{14}$, we use $2^{30}$ pairs to generate $S_{i+1} \oplus S_{i+1}^{\prime}$, then compute $p_{14,0}^{17}$ and $p_{14,1}^{17}$. From the simulation, we found that $p_{14,0}^{17}=0.50227$ and $p_{14,1}^{17}=0.50117$. We denote the average of $p_{14,0}^{17}$ and $p_{14,1}^{17}$ as $\bar{p}_{14}^{17}$, i.e., $\bar{p}_{14}^{17}=\frac{p_{14,0}^{17}+p_{14,1}^{17}}{2}=0.50172$. Running a similar simulation, we found that $p_{13,0}^{17}=0.50175$ and $p_{13,1}^{17}=0.50169$. For all the $j \neq 13, j \neq 14$, we found that $p_{j, 0}^{17} \approx \bar{p}_{14}^{17}$ and $p_{j, 1}^{17} \approx \bar{p}_{14}^{17}$. The value of $X_{i+1,0}^{15} \oplus X_{i+1,0}^{14}$ is recovered as follows: from the keystreams, we compute the fraction for which $S_{i+1}^{17} \oplus S_{i+1}^{\prime 17}=0$. If it is larger than $\bar{p}_{14}^{17}$, then the value of $X_{i+1,0}^{15} \oplus X_{i+1,0}^{14}$ is considered to be 0 ; otherwise the value of $X_{i+1,0}^{15} \oplus X_{i+1,0}^{14}$ is considered to be 1 .

We now compute the number of plaintext pairs required to determine the value of $X_{i+1,0}^{15} \oplus X_{i+1,0}^{14}$. Suppose that $N$ pairs of plaintexts are used. The standard deviation is $\sigma=\sqrt{N \times \bar{p}_{14}^{17} \times\left(1-\bar{p}_{14}^{17}\right)}$. To determine the value of $X_{i+1,0}^{15} \oplus X_{i+1,0}^{14}$ with success rate 0.99 , we require that $N \times\left(\left(p_{14,0}^{17}-p_{14,1}^{17}\right)-\left(p_{13,0}^{17}-p_{13,1}^{17}\right)\right)>$ $4.66 \times \sigma$ (The cumulative distribution function of the normal distribution gives value 0.99 at the point $2.33 \sigma)$. Thus we require that $N>2^{22.27}$.

We used the Phelix $C$ source code submitted to eSTREAM in the experiments 1

Experiment 1. The goal of this experiment is to recover the value of $X_{1,0}^{15} \oplus X_{1,0}^{14}$. Each plaintext has two words $P_{0}$ and $P_{1}$. For each plaintext pair, the two words differ only in the least significant bit of $P_{0} . N$ plaintext pairs are used for each key to determine the value of $X_{1,0}^{15} \oplus X_{1,0}^{14}$ as follows: if the fraction of cases for

${ }^{1}$ Note that we have found and corrected a small bug in this source code. The output should be computed as $S_{i}=Y_{4}^{(i)}+Z_{4}^{(i-4)}$ in stead of $S_{i}=Y_{4}^{(i)}+Z_{4}^{(i-3)}$. 
which $S_{1}^{17} \oplus S_{1}^{\prime 17}=0$ is larger than $\bar{p}_{14}^{17}=0.50172$, then the value of $X_{1,0}^{15} \oplus X_{1,0}^{14}$ is considered to be 0 ; otherwise the value of $X_{1,0}^{15} \oplus X_{1,0}^{14}$ is considered to be 1 . A random nonce was used for each plaintext pair. We tested 200 keys in the experiment. For $N=2^{22.3}$, the values of $X_{1,0}^{15} \oplus X_{1,0}^{14}$ of 183 keys are determined correctly. For $N=2^{25}$, the values of $X_{1,0}^{15} \oplus X_{1,0}^{14}$ of 192 keys are determined correctly.

Experiment 1 shows that the value of $X_{1,0}^{15} \oplus X_{1,0}^{14}$ can be determined successfully by introducing a difference in the least significant bit of $P_{0}$, but with a higher error rate. The reason is that other bits of $X_{1,0}$ affects the determination of $X_{1,0}^{15} \oplus X_{1,0}^{14}$ in a subtle way.

We now proceed to recover the other bits of $X_{1,0}$. By rotating the one-bit difference between $P_{0}$ and $P_{0}^{\prime}$, and using the same threshold value, we can determine the value of $X_{1,0}^{j+1} \oplus X_{1,0}^{j}$ for $2 \leq j \leq 3,5 \leq j \leq 10$ and $14 \leq j \leq 28$.

Thus we are able to recover 23 bits of information on each $X_{i, 0}$. Note that the 256 -bit key is recovered from eight consecutive $X_{i, 0}$. Thus we are able to recover $23 \times 8=184$ bits of the key with success rate about $\frac{192}{200}=0.96$. The number of plaintext pairs required in the attack is about $2^{25} \times 32 \times 8=2^{33}$.

We need to improve the above attack in two approaches: recovering more key bits and improving the success rate. The direct approach is to adjust the threshold value for each key bit position. In the following, we illustrate a more advanced approach which recovers the values of $Z_{4}^{(i)}$ before recovering the key.

\section{Improving the Attack on Phelix}

In the above attack, we use a random nonce for each plaintext pair, i.e., every nonce is used twice with the same key. When the nonce is used many times with the same key, we can introduce the difference at $P_{i}$ and recover the value of $Z_{4}^{i-3}$ by observing the distribution of $S_{i+1} \oplus S_{i+1}^{\prime}$. Then we proceed to recover $X_{i+1,0}$.

\subsection{Recovering $Z_{4}^{(i)}$}

We introduce the difference to the least significant bit of $P_{i}\left(P_{i} \oplus P_{i}^{\prime}=1\right)$. A simulation is carried out to determine the distribution of $Y_{4}^{(i+1)} \oplus Y_{4}^{\prime(i+1)}$. We use the randomly generated $Y_{k}^{(i)}(4 \geq k \geq 0), P_{i}, X_{i, 1}, X_{i+1,0}$ in the simulation. Denote $\dot{p}_{n}$ as the probability that $Y_{4}^{(i+1), n} \oplus Y_{4}^{\prime(i+1), n}=0$. With $2^{30}$ pairs, we obtain the values of $\dot{p}_{n}$ in Table 2 .

From Table 2, we notice that $Y_{4}^{(i+1)} \oplus Y_{4}^{\prime(i+1)}$ is heavily biased. For example, $Y_{4}^{(i+1), 2}=Y_{4}^{\prime(i+1), 2}$ with probability about 0.70291 , while $Y_{4}^{(i+1), 3}=Y_{4}^{\prime(i+1), 3}$ with probability about 0.22246 . Note that $S_{i+1}=Y_{4}^{(i+1)} \oplus Z_{4}^{(i-3)}$, according to Theorem 2, the distribution of $S_{i+1} \oplus S_{i+1}^{\prime}$ is affected by the value of $Z_{4}^{(i-3), 3} \oplus$ $Z_{4}^{(i-3), 2}$. Next we carry out simulations to characterize this relation.

We use the randomly generated $Y_{k}^{(i)}(4 \geq k \geq 0), P_{i}, X_{i, 1}, X_{i+1,0}, Z_{4}^{(i-3)}$ in the simulation. The one-bit difference is introduced to $P_{i}$, i.e., $P_{i} \oplus P_{i}^{\prime}=2^{j}$. 
Table 2. The probability that $Y_{4}^{(i+1), j} \oplus Y_{4}^{\prime(i+1), j}=0$ for $P_{i} \oplus P_{i}^{\prime}=1$

\begin{tabular}{|c|r||c|r||c|r||c|r|}
\hline$j$ & $\dot{p}_{j}-0.5$ & $j$ & $\dot{p}_{j}-0.5$ & $j$ & $\dot{p}_{j}-0.5$ & $j$ & $\dot{p}_{j}-0.5$ \\
\hline 0 & 0.03326 & 8 & 0.00003 & 16 & -0.00003 & 24 & 0.00046 \\
1 & 0.12983 & 9 & 0.03517 & 17 & 0.00268 & 25 & 0.05926 \\
2 & 0.20291 & 10 & 0.00002 & 18 & -0.00001 & 26 & 0.15064 \\
3 & -0.27754 & 11 & 0.00001 & 19 & -0.00266 & 27 & -0.24028 \\
4 & -0.00005 & 12 & 0.00000 & 20 & -0.00004 & 28 & 0.00001 \\
5 & 0.05663 & 13 & 0.02293 & 21 & 0.02276 & 29 & 0.05770 \\
6 & -0.15327 & 14 & -0.00001 & 22 & 0.07434 & 30 & 0.15508 \\
7 & -0.00001 & 15 & -0.00001 & 23 & -0.14414 & 31 & -0.24907 \\
\hline
\end{tabular}

Denote $\ddot{p}_{j, 0}^{n}$ as the probability that $S_{i+1}^{n} \oplus S_{i+1}^{\prime n}=0$ when $Z_{4}^{(i-3), j+1} \oplus Z_{4}^{(i-3), j}=0$. And denote $\ddot{p}_{j, 1}^{n}$ as the probability that $S_{i+1}^{n} \oplus S_{i+1}^{\prime n}=0$ when $Z_{4}^{(i-3), j+1} \oplus$ $Z_{4}^{(i-3), j}=1$. For each value of $Z_{4}^{(i-3), 3}$ and $Z_{4}^{(i-3), 2}$, we use $2^{28}$ plaintext pairs. We find that $\ddot{p}_{2,0}^{5}=0.5461$ and $\ddot{p}_{2,1}^{5}=0.5193$. The large difference between $\ddot{p}_{2,0}^{5}$ and $\ddot{p}_{2,1}^{5}$ shows that the value of $Z_{4}^{(i-3), 3} \oplus Z_{4}^{(i-3), 2}$ can be determined with success rate 0.999 with about $2^{13.9}$ plaintext pairs (The cumulative distribution function of the normal distribution gives value 0.999 at the point $3.1 \sigma$ ).

The above approach is able to recover $Z_{4}^{(0)}$, but the success rate is not that high according to our experiment. In the following, we use a new approach to determine $Z_{4}^{(0)}$. To reduce the interference between the bits of $Z_{4}^{(i-3)}$, we recover the least significant bit of $Z_{4}^{(i-3)}$ first, then proceed to recover the more significant bits bit-by-bit.

We start with determining the value of $Z_{4}^{(i-3), 0}$. Let $P_{i} \oplus P_{i}^{\prime}=1$. Running the simulation with2 $2^{28}$ plaintext pairs, we found that $\ddot{p}_{-1,0}^{2}=0.70296$, and $\ddot{p}_{-1,1}^{2}=0.65422\left(\right.$ let $Z_{4}^{(i-3),-1}=0$ ). To determine the value of $Z_{4}^{(i-3), 0}$ with success rate 0.999 , we need about $2^{12.0}$ plaintext pairs.

Experiment 2. The goal of this experiment is to determine the value of $Z_{4}^{(0), 0}$. Each plaintext has five random words $P_{i}(0 \leq i \leq 4)$. For each plaintext pair, the difference is only in the least significant bit of $P_{3} . N$ plaintext pairs are used for each key/nonce pair to determine the value of $Z^{(0), 0}$ as follows: if the rate that $S_{4}^{2} \oplus S_{4}^{\prime 2}=0$ is larger than $\frac{\ddot{p}_{-1,0}^{2}+\ddot{p}_{-1,1}^{2}}{2}=\frac{0.70296+0.65422}{2}=0.6786$, then the value of $Z_{4}^{(0), 0}$ is considered to be 0 ; otherwise the value of $Z_{4}^{(0), 0}$ is considered to be 1 . We tested $1000 \mathrm{key} /$ nonce pairs in the experiment. For $N=2^{12}$, the values of $Z_{4}^{(0), 0}$ of $998 \mathrm{key} /$ nonce pairs are determined correctly. For $N=2^{13}$, the values of $Z_{4}^{(0), 0}$ of all the key/nonce pairs are determined correctly.

After recovering the value of $Z_{4}^{(i-3), 0}$, we proceed to recover the values of the other bits of $Z_{4}^{(i-3)}$. Let $Z_{4}^{(i-3),(n-1 \cdots 0)}$ denote the $n$ least significant bits of $Z^{(i-3)}$, i.e., $Z_{4}^{(i-3),(n-1 \cdots 0)}=Z^{(i-3)} \bmod 2^{n}$. Let the difference be introduced to the $k$ th least significant bit of $P_{i}$, i.e., $P_{i} \oplus P_{i}^{\prime}=2^{k}$. Denote $\grave{p}_{Z_{4}^{(i-3),(n-1 \cdots 0)}}^{k, j, 0}$ as 
the probability that the value of the $j$ th bit of $\left(S_{i+1}-Z_{4}^{(i-3),(n-1 \cdots 0)}\right) \oplus\left(S_{i+1}^{\prime}-\right.$ $\left.Z_{4}^{(i-3),(n-1 \cdots 0)}\right)$ is 0 when $Z_{4}^{(i-3), n}=0$. Denote $\dot{p}_{Z_{4}^{(i-3),(n-1 \cdots 0)}}^{k, j, 1}$ as the probability that the value of the $j$ th bit of $\left(S_{i+1}-Z_{4}^{(i-3),(n-1 \cdots 0)}\right) \oplus\left(S_{i+1}^{\prime}-Z_{4}^{(i-3),(n-1 \cdots 0)}\right)$ is 0 when $Z_{4}^{(i-3), n}=1$. If the value of $Z_{4}^{(i-3),(n-1 \cdots 0)}$ is determined correctly, then $\grave{p}_{Z_{4}^{(i-3),(n-1 \cdots 0)}}^{k, j, 0}=\grave{p}_{0}^{k, j, 0}$, and $\grave{p}_{Z_{4}^{(i-3),(n-1 \cdots 0)}}^{k, j, 1}=\grave{p}_{0}^{k, j, 1}$. This property is important for recovering $Z_{4}^{(i-3)}$.

Let $P_{i} \oplus P_{i}^{\prime}=2$. We use $2^{28}$ plaintext pairs in the simulation. We found that $\grave{p}_{0}^{1,3,0}=0.66469$ and $\grave{p}_{0}^{1,3,1}=0.60220$. It shows that when $Z_{4}^{(i-3), 0}=0$, if the rate that $S_{i+1}^{3} \oplus S_{i+1}^{\prime 3}=0$ is larger than $\frac{0.66469+0.60220}{2}=0.63345$, then the value of $Z_{4}^{(i-3), 1}$ is determined to be 0 ; otherwise the value of $Z_{4}^{(i-3), 1}$ is determined to be 1 . We need about $2^{11.3}$ plaintext pairs to determine the value of $Z_{4}^{(i-3), 1}$ correctly with success rate 0.999 . Using the Phelix code in the experiment, we tested 1000 random key/nonce pairs satisfying $Z_{4}^{(0), 0}=0$, and $2^{12}$ plaintext pairs are used for each key/nonce pair with the difference $P_{3} \oplus P_{3}^{\prime}=2$. We found that all the 1000 values of $Z_{4}^{(0), 1}$ are determined correctly. If $Z_{4}^{(i-3), 0}=1$, we observe the third least significant bit of $\left(S_{i+1}-1\right) \oplus\left(S_{i+1}^{\prime}-1\right)$, and we can determine the value of $Z_{4}^{(0), 1}=0$ with success rate 0.999 with about $2^{11.3}$ plaintext pairs.

Let $P_{i} \oplus P_{i}^{\prime}=2^{2}$, we are able to determine the value of $Z_{4}^{(i-3), 2}$ by observing the fourth least significant bit of $\left(S_{i+1}-Z_{4}^{(i-3),(1 \cdots 0)}\right) \oplus\left(S_{i+1}^{\prime}-Z_{4}^{(i-3),(1 \cdots 0)}\right)$. In general, let $P_{i} \oplus P_{i}^{\prime}=2^{j}$, then we are able to determine the value of $Z_{4}^{(i-3), j}$ by observing the $(j+2)$ th least significant bit of $\left(S_{i+1}-Z_{4}^{(i-3),(j-1 \cdots 0)}\right) \oplus\left(S_{i+1}^{\prime}-\right.$ $\left.Z_{4}^{(i-3),(j-1 \cdots 0)}\right)$ with about $2^{12}$ plaintext pairs. Thus we are able to recover $Z_{4}^{(i-3)}$ (except the values of $Z_{4}^{(i-3), 30}$ and $Z_{4}^{(i-3), 31}$ ) with success rate very close to 1 . The number of plaintext pairs required in the above attack is about $2^{12} \times 30 \approx 2^{17}$.

\subsection{Recovering $X_{i+1,0}$}

After recovering $Z_{4}^{(i-3)}$ (except $Z_{4}^{(i-3), 31}$ and $Z_{4}^{(i-3), 30}$ ), we know the value of $\left(S_{i+1}-Z_{4}^{(i-3),(29 \cdots 0)}\right) \oplus\left(S_{i+1}^{\prime}-Z_{4}^{(i-3),(29 \cdots 0)}\right)$. Thus we know the value of $Y^{(i+1), j} \oplus Y^{\prime(i+1), j}(0 \leq j \leq 30)$. Then we are able to recover $X_{i+1,0}$ more efficiently.

Let two plaintexts differ only in the $i$ th word. And let $P_{i} \oplus P_{i}^{\prime}=1$. We use the randomly generated $Y_{k}^{(i)}(4 \geq k \geq 0), P_{i}, X_{i, 1}, X_{i+1,0}, Z_{4}^{(i-3)}$ in the simulation. For every value of the two bits $X_{i, 0}^{j+1}$ and $X_{i, 0}^{j}$, we use $2^{28}$ plaintext pairs to generate $Y_{4}^{(i+1)} \oplus Y_{4}^{\prime(i+1)}$, then compute $p_{j, 0}^{n}$ and $p_{j, 1}^{n}$ (suppose that $S_{i+1}=Y_{4}^{(i+1)}$ since $Z_{4}^{(i-3)}$ is known). Thus $N=2^{29}$, and $\sigma=2^{13.5}$. We list the following two large biases $\Delta \tilde{p}_{j}^{n}$ :

For $j=9, \Delta \tilde{p}_{9}^{13}=144.1$

For $j=10, \Delta \tilde{p}_{10}^{13}=362.12$ 
We use $\Delta \tilde{p}_{9}^{13}$ and $\Delta \tilde{p}_{10}^{13}$ in the attack. Note that the values of $X_{i+1,0}^{10} \oplus X_{i+1,0}^{9}$ and $X_{i+1,0}^{11} \oplus X_{i+1,0}^{10}$ both affect the distribution of $Y_{4}^{(i+1), 13} \oplus Y_{4}^{\prime(i+1), 13}$. We carried out a simulation with $2^{30}$ chosen plaintext pairs to determine how the value of $X_{i+1,0}^{9}$ affects the value of $Y_{4}^{(i+1), 13}$. If $X_{i+1,0}^{9}=0$, and the values of $X_{i+1,0}^{11} \| X_{i+1,0}^{0}$ are $00,11,01$ and 11 (in binary format), we obtain that $Y_{4}^{(i+1), 13} \oplus Y_{4}^{\prime(i+1), 13}=0$ with probability $0.53033,0.52334,0.51946$ and 0.51864 , respectively; if $X_{i+1,0}^{9}=1$, we obtain that $Y_{4}^{(i+1), 13} \oplus Y_{4}^{\prime(i+1), 13}=0$ with probability $0.52334,0.53030,0.51861$ and 0.51948 , respectively. We thus let $p_{0,0}^{13}=0.52334$, and $p_{0,1}^{13}=\frac{0.51946+0.51948}{2}=0.51947$. About $2^{19.3}$ plaintext pairs are required to determine the value of $X_{i+1,0}^{11} \oplus X_{i+1,0}^{10}$ with success rate 0.999 .

Experiment 3. Suppose that the value of $Z_{4}^{(0)}$ is known. The goal of this experiment is to determine the value of $X_{4,0}^{11} \oplus X_{4,0}^{10}$. Each plaintext has five random words $P_{i}(0 \leq i \leq 4)$. For each plaintext pair, those five words differ only in the least significant bit of $P_{3} . N$ plaintext pairs are used for each key/nonce pair to determine the value of $X_{4,0}^{11} \oplus X_{4,0}^{10}$ as follows: if the rate that $Y_{4}^{13} \oplus Y_{4}^{\prime 13}=0$ is larger than $\frac{0.52334+0.51947}{2}=0.52140$, then the value of $X_{4,0}^{11} \oplus X_{4,0}^{10}$ is considered to be 0 ; otherwise the value of $X_{4,0}^{11} \oplus X_{4,0}^{10}$ is considered to be 1 . We tested 1000 key/nonce pairs in the experiment. For $N=2^{19.3}, 948$ values of $1000 X_{4,0}^{11} \oplus X_{4,0}^{10}$ are determined correctly. We change the threshold value 0.52140 to 0.52035 , then 970 values of $1000 X_{4,0}^{11} \oplus X_{4,0}^{10}$ are determined correctly for $N=2^{20}, 976$ values are determined correctly for $N=2^{21}, 990$ values are determined correctly for $N=2^{22}$.

Experiment 3 shows that the value of $X_{4,0}^{11} \oplus X_{4,0}^{10}$ can be determined successfully by introducing a difference in the least significant bit of $P_{3}$. With $2^{22}$ chosen pairs, we are able to determine the value of $X_{4,0}^{11} \oplus X_{4,0}^{10}$ with success rate about 0.99 .

Then we shift the one-bit difference to recover the values of $X_{1,0}^{j+1} \oplus X_{1,0}^{j}$ for $2 \leq j \leq 28$. The threshold value needs to be modified for different values of $j$. The results are given in Table 3 in Appendix A. Note that according to Experiment 3, the threshold values should be slightly adjusted to achieve high success rate.

The reason that the values of $X_{4,0}^{j+1} \oplus X_{4,0}^{j}$ cannot be recovered for $j \geq 29$ is that the value of $X_{1,0}^{j+1} \oplus X_{1,0}^{j}$ cannot affect the distribution of $S_{1}^{j+3} \oplus S_{1}^{\prime j+3}$ since $S_{1} \oplus S_{1}^{\prime}$ is a 32-bit word. The reason that the number of plaintext required for $j=9$ is relatively small is that the difference for $j=13$ is introduced to the most significant bit of the word $P_{3}$, thus it causes less difference propagation, and results in a larger bias in the keystream.

Note that the most significant bit of $Y_{4}^{(i+1)} \oplus Y_{4}^{\prime(i+1)}$ is not known since $Z_{4}^{i-1,31}$ and $Z_{4}^{i-1,30}$ are not recovered. Thus to determine the value of $X_{1,0}^{29} \oplus X_{1,0}^{28}$, we 
need to consider the most significant bit of $\left(S_{i+1}-Z_{4}^{(i-3),(29 \cdots 0)}\right) \oplus\left(S_{i+1}^{\prime}-\right.$ $\left.Z_{4}^{(i-3),(29 \cdots 0)}\right)$. The threshold value needs to be changed to 0.51128 ; and the number of plaintext pairs required is $2^{22.1}$.

After recovering the values of $X_{1,0}^{j+1} \oplus X_{1,0}^{j}$ for $2 \leq j \leq 28$, we proceed to determine the value of $X_{i+1,0}^{0}, X_{i+1,0}^{1}$ and $X_{i+1,0}^{2}$.

We start with recovering $X_{i+1,0}^{0}$. Let $P_{i} \oplus P_{i}^{\prime}=2^{21}$. Running the simulation with $2^{28}$ plaintext pairs, we found that $p_{21,0}^{2}=0.51596, p_{21,1}^{2}=0.50355$. Thus $2^{15.93}$ plaintext pairs are needed to determine the value of $X_{i+1,0}^{0}$ with success rate 0.999 . Using the Phelix code in the experiment, we introduce the difference $P_{3} \oplus P_{3}^{\prime}=2^{21}$, and set the threshold value as $\frac{0.51596+0.50355}{2}=0.50975$. We tested $1000 \mathrm{key} /$ nonce pairs in the experiment. With $2^{16}$ plaintext pairs, all the values of the $1000 X_{4,0}^{0}$ are determined correctly.

After determine the value of $X_{i+1,0}^{0}$, we determine the value of $X_{i+1,0}^{1}$ as follows. The simulation shows that the value of $X_{i+1,0}^{1}$ can be determined only when $X_{i+1,0}^{0}=0$. For $X_{i+1,0}^{0}=0$, we set the difference as $P_{i} \oplus P_{i}^{\prime}=2^{22}$. With $2^{28}$ chosen plaintext pairs, we found that if $X_{i+1,0}^{1}=0$, then $Y_{4}^{(i+1), 3}=0$ with rate 0.51528 ; otherwise $Y_{4}^{(i+1), 3}=0$ with rate 0.50459 . With $2^{16.4}$ plaintext pairs, the value of $X_{i+1,0}^{1}$ can be determined with success rate 0.999 . Using the Phelix code in the experiement, we introduce the difference $P_{3} \oplus P_{3}^{\prime}=2^{22}$, and set the threshold value as $\frac{0.51528+0.50459}{2}=0.50994$. We tested $1000 \mathrm{key} /$ nonce pairs with $X_{4,0}^{0}=0$ in the experiment. With $2^{16.4}$ plaintext pairs, all the values of the $1000 X_{4,0}^{1}$ are determined correctly. It shows that the value of $X_{i+1,0}^{1}$ can be determined successfully if $X_{4,0}^{0}=0$.

We continue to recover the value of $X_{i+1,0}^{2}$. We introduce a difference to the 15 th least significant bit of $P_{i}$, and observe the distribution of $Y_{4}^{(i-3), 4}$. We carry out a simulation with $2^{31}$ plaintext pairs with $P_{3} \oplus P_{3}^{\prime}=2^{15} .2^{31}$ plaintext pairs are used for each value of $X_{i+1,0}^{1} X_{i+1,0}^{0}$. When $X_{i+1,0}^{0}=0$, if $X_{i+1,0}^{1}=0$, the fraction of values for which $Y_{4}^{(i-3), 4}=0$ if $X_{i+1,0}^{2}=0$ and $X_{i+1,0}^{2}=1$ are 0.53106 and 0.52613 , respectively; if $X_{i+1,0}^{1}=1$, the franction of values for which $Y_{4}^{(i-3), 4}=0$ if $X_{i+1,0}^{2}=0$ and $X_{i+1,0}^{2}=1$ are 0.52318 and 0.52315 , respectively. It shows that the value $X_{i+1,0}^{2}$ can only be determined if the values of $X_{i+1,0}^{1}$ and $X_{i+1,0}^{1}$ are both zero, and $2^{18.6}$ plaintext pairs are required to achieve the success rate 0.999 .

In the above attacks, we recovered 28.75 bits of $X_{i+1,0}: X_{i+1,0}^{j+1} \oplus X_{i+1,0}^{j}$ for $2 \leq j \leq 28, X_{i+1,0}^{0}, X_{i+1,0}^{1}$ (only if $\left.X_{i+1,0}^{0}=0\right)$, and $X_{i+1,0}^{1}$ (only if $X_{i+1,0}^{0}=0$ and $\left.X_{i+1,0}^{1}=0\right) .27$ bits of $X_{i+1,0}^{j+1} \oplus X_{i+1,0}^{j}(2 \leq j \leq 28)$ can be determined according to Table 3. From Experiment 3, we know that if we slightly adjust the threshold value, and use about $2^{2.7}$ times the number of plaintext pairs compared to Table 3 , the success rate is about 0.99 . The number of plaintext pairs required to determine these 27 bits is thus about $27 \times 2^{22.2} \times 2^{2.7}=2^{29.7}$. The number of plaintext pairs to determine $X_{i+1,0}^{0}, X_{i+1,0}^{1}$ and $X_{i+1,0}^{2}$ is small compared to 
$2^{29.7}$. The attack to recover 28.75 bits of $X_{i+1,0}$ requires thus about $2^{32.7}$ chosen plaintext pairs.

After recovering eight consecutive $X_{i+1,0}$, we recovered $28.75 \times 8=230$ key bits. To recover the 256-bit key, the amount of operations required is about $2^{256-230+\left(\begin{array}{c}27 \times 8 \\ 0.01 \times 27 \times 8\end{array}\right)}=2^{41.5}$.

The number of chosen plaintext pairs required in the attack is about $2^{29.7} \times 8=$ $2^{32.7}$. The length of each plaintext ranges from 5 to 13 words. Thus the total amount of chosen plaintext required is about $2 \times 2^{32.7} \times \frac{5+13}{2} \approx 2^{37}$ words. (The number of plaintext pairs needed to recover 8 consecutive $Z_{4}^{(i)}$ is about $2^{17} \times 8=2^{25}$. It is small compared to $2^{32.7}$ ).

\section{An Approach to Strengthen Helix and Phelix}

In Helix and Phelix, the plaintext is used to affect the internal state of the cipher. In order to achieve a high encryption speed, each plaintext word affects the keystream without passing through sufficient confusion and diffusion layers. This is the intrinsic weakness in the structure of Helix and Phelix. In the following, we provide a method to reduce the effect of such weakness.

The security of the encryption of Helix and Phelix can be improved significantly if a secure one-way function is used to generate the initial state of the cipher from the key and nonce. Then even if the internal state of one particular nonce is recovered, the impact on the security of the encryption is very limited since the key of the cipher is not affected. We believe that such an approach can be applied to improve the security of all the ciphers that use the plaintext to affect the internal state.

However, we must point out that such an approach does not substantially improve the security of the MAC in Helix and Phelix. Once an internal state is recovered, the attacker can forge many messages related to that particular nonce.

\section{Conclusion}

Phelix is vulnerable to a key recovery attack when chosen nonces and chosen plaintexts are used. The computational complexity of the attack is much less than that of the attack against Helix. Our attack shows that Phelix fails to strengthen Helix in this respect.

We believe that one necessary requirement for a secure general-purpose stream cipher is that the key of the cipher should not be recoverable even if the attacker can control the generation of the nonce. In practice an attacker may gain access to a Phelix encryption device for a while, reuse a nonce and recover the key. We thus consider Phelix as insecure. (When the integrity checking mechanism is not enforced, an attacker can even modify the nonces in ciphertext and obtain repeated nonces.) Muller has pointed out the practical impact of the key recovery attack with reused nonces on the security of Helix in detail 2. Muller stated 
clearly the difference between the nonce reusing attack against Helix and that against a synchronous stream cipher since the attack against Helix results in key recovery. The same comments apply to the attacks against Phelix.

\section{Acknowledgements}

The authors would like to thank the anonymous reviewers of FSE 2007 for their helpful comments.

\section{References}

1. Ferguson, N., Whiting, D., Schneier, B., Kelsey, J., Lucks, S., Kohno, T.: Helix, Fast Encryption and Authentication in a Single Cryptographic Primitive. In: Johansson, T. (ed.) FSE 2003. LNCS, vol. 2887, pp. 330-346. Springer, Heidelberg (2003)

2. Muller, F.: Differential Attacks against the Helix Stream Cipher. In: Roy, B., Meier, W. (eds.) FSE 2004. LNCS, vol. 3017, pp. 94-108. Springer, Heidelberg (2004)

3. Paul, S., Preneel, B.: Solving Systems of Differential Equations of Addition. In: Boyd, C., González Nieto, J.M. (eds.) ACISP 2005. LNCS, vol. 3574, pp. 75-88. Springer, Heidelberg (2005)

4. Paul, S., Preneel, B.: Near Optimal Algorithms for Solving Differential Equations of Addition with Batch Queries. In: Maitra, S., Madhavan, C.E.V., Venkatesan, R. (eds.) INDOCRYPT 2005. LNCS, vol. 3797, pp. 90-103. Springer, Heidelberg (2005)

5. Whiting, D., Schneier, B., Lucks, S., Muller, F.: Phelix: Fast Encryption and Authentication in a Single Cryptographic Primitive. eSTREAM, ECRYPT Stream Cipher Project Report 2005/027 (2005)

6. Wu, H., Preneel, B.: Cryptanalysis of the Stream Cipher ABC v2. In: Selected Areas in Cryptography - SAC 2006. LNCS (to appear)

\section{A The Complexity to Recover $X_{i+1,0}$ with $Z_{4}^{(i-3)}$ Known}

The number of plaintext pairs and the threshold value required to recover the value of each $X_{i+1,0}^{j+1} \oplus X_{i+1,0}^{j}(2 \leq j \leq 28)$ are given in Table 3 . Each value $n$ in the second column indicates that the difference is introduced in the $n$th least significant bit of $P_{i}$. Each value $n$ in the third column shows that the $n$th least significant bit of $Y_{4}^{(i+1)}$ is used in the attack. 
Table 3. The number of plaintext pairs for recovering $X_{i+1,0}^{j+1} \oplus X_{i+1,0}^{j}$

\begin{tabular}{|c|c|c|c|c|}
\hline$j$ & $\begin{array}{l}\text { Difference } \\
\text { position } \\
\text { in } P_{i}\end{array}$ & $\begin{array}{c}\text { Bit } \\
\text { position } \\
\text { in } Y_{4}^{(i+1)}\end{array}$ & $\begin{array}{c}\text { Threshold } \\
\text { value }\end{array}$ & $\begin{array}{c}\text { Plaintext } \\
\text { Pairs }\end{array}$ \\
\hline 2 & 24 & 5 & 0.51101 & $2^{24.4}$ \\
\hline 3 & 25 & 6 & 0.51110 & $2^{23.1}$ \\
\hline 4 & 26 & 7 & 0.51120 & $2^{22.5}$ \\
\hline 5 & 27 & 8 & 0.51125 & $2^{22.3}$ \\
\hline 6 & 28 & 9 & 0.51091 & $2^{22.4}$ \\
\hline 7 & 29 & 10 & 0.51116 & $2^{23.6}$ \\
\hline 8 & 30 & 11 & 0.51562 & $2^{20.7}$ \\
\hline 9 & 31 & 12 & 0.54353 & $2^{18.4}$ \\
\hline 10 & 0 & 13 & 0.52141 & $2^{19.3}$ \\
\hline 11 & 1 & 14 & 0.52099 & $2^{19.3}$ \\
\hline 12 & 2 & 15 & 0.51850 & $2^{19.5}$ \\
\hline 13 & 3 & 16 & 0.50998 & $2^{21.3}$ \\
\hline 14 & 4 & 17 & 0.51107 & $2^{21.9}$ \\
\hline 15 & 5 & 18 & 0.51128 & $2^{22.2}$ \\
\hline 16 & 6 & 19 & 0.51129 & $2^{22.2}$ \\
\hline 17 & 7 & 20 & 0.51131 & $2^{22.2}$ \\
\hline 18 & 8 & 21 & 0.51128 & $2^{22.1}$ \\
\hline 19 & 9 & 22 & 0.51117 & $2^{21.7}$ \\
\hline 20 & 10 & 23 & 0.51149 & $2^{22.2}$ \\
\hline 21 & 11 & 24 & 0.51172 & $2^{22.0}$ \\
\hline 22 & 12 & 25 & 0.51187 & $2^{22.0}$ \\
\hline 23 & 13 & 26 & 0.51191 & $2^{22.0}$ \\
\hline 24 & 14 & 27 & 0.51185 & $2^{22.1}$ \\
\hline 25 & 15 & 28 & 0.51129 & $2^{22.2}$ \\
\hline 26 & 16 & 29 & 0.51129 & $2^{22.1}$ \\
\hline 27 & 17 & 30 & 0.51131 & $2^{22.2}$ \\
\hline 28 & 18 & 31 & 0.51130 & $2^{22.1}$ \\
\hline
\end{tabular}

